\title{
Quantum mechanical effects analysis of nanostructured solar cell models
}

\author{
Andrei Badea $^{1}$, Florin Dragan ${ }^{1, *}$, Laurentiu Fara ${ }^{1,2}$, and Paul Sterian ${ }^{1,2}$ \\ 1 Academic Center for Optical Engineering and Photonics, Faculty of Applied Sciences, University "Politehnica" of Bucharest, 313 \\ Splaiul Independentei, 060042 Bucharest, Romania \\ 2 Academy of Romanian Scientists, 54 Splaiul Independentei, 050094 Bucharest, Romania
}

\begin{abstract}
The quantum mechanical effects resulted from the inclusion of nanostructures, represented by quantum wells and quantum dots, in the i-layer of an intermediate band solar cell will be analyzed. We will discuss the role of these specific nanostructures in the increasing of the solar cells efficiency. InAs quantum wells being placed in the i-layer of a gallium arsenide (GaAs) p-i-n cell, we will analyze the quantum confined regions and determine the properties of the eigenstates located therein. Also, we simulate the electroluminescence that occurs due to the nanostructured regions.
\end{abstract}

\section{Introduction}

In the last few years, solar energy has become a field of great interest, as the need for alternative energy systems have become increasingly important. Some current research efforts are focused on improving photovoltaic conversion efficiency by using low-dimensional nanostructures. More specific to this work, incorporating nanostructures like quantum dots in an otherwise conventional III-V PV device has been proposed as a viable method for increasing the conversion efficiency [1].

A nanostructure can be considered as a structure with at least one spatial dimension small enough for the quantum confinement effects to become significant. The quantum well can be thought as a classic example of a nanostructure [2]. In this example, the material is enclosed in one spatial dimension while the other two dimensions are bulk-sized. One way to achieve such a structure is to grow a thin film of a semiconductor material in-between two other bulk-sized semiconductors. This structure is shown in Figure 1, where the bulk regions are made of the same material, which is different from the quantum well material. In addition, the quantum well material should display a smaller bandgap than the bulk material; the associated energy band diagram is presented in Figure 2.

Another well-known low-dimensional nanostructure is the quantum dot. Unlike quantum well, the quantum dot can be seen as a zero-dimensional structure. The excitons are confined in all three spatial dimensions,

\footnotetext{
* e-mail: florin.dragan@sdettib.pub.ro
}

thus leading to optical and electrical properties that prove useful in biomedical imaging, as well as the possibility for use in electronic devices and solar energy systems. A single quantum dot placed within a bulk material would exhibit a similar band diagram as in Figure 2 [3].

The quantum well PV cells were first proposed in 1990 by the research group of professor Keith Barnham [4], based on the idea that the use of the quantum wells could improve the photovoltaic cells by extending their spectral response, as well as by increasing the photocurrent. One year later, this idea was experimentally proved by using a $\mathrm{GaAs} / \mathrm{Al}_{\mathrm{x}} \mathrm{Ga}_{1-\mathrm{x}} \mathrm{As}$ multi-layered structure [5].

The objective of this paper is to offer a few modeling solutions necessary for the analysis and efficiency improvement of the next generation of PV devices, by studying the quantum mechanical effects involved.

\section{Materials and method}

\subsection{Materials}

Gallium arsenide (GaAs) is an important semiconductor material for high-cost, high-efficiency solar cells. It is used for single-crystalline thin film solar cells and also for multi-junction solar cells. GaAs-based devices hold the world record for the highest-efficiency single-junction solar cell at $28.8 \%$ [6]. This high efficiency is attributed to the extreme high quality GaAs epitaxial growth and the promotion of photon recycling by the thin film design [7].

The quantum well solar cell is a p-i-n structure, which contains quantum wells built in the intrinsic [8]. The device discussed in this work is a simple GaAs p-i-n solar cell as shown in Figure 3. 


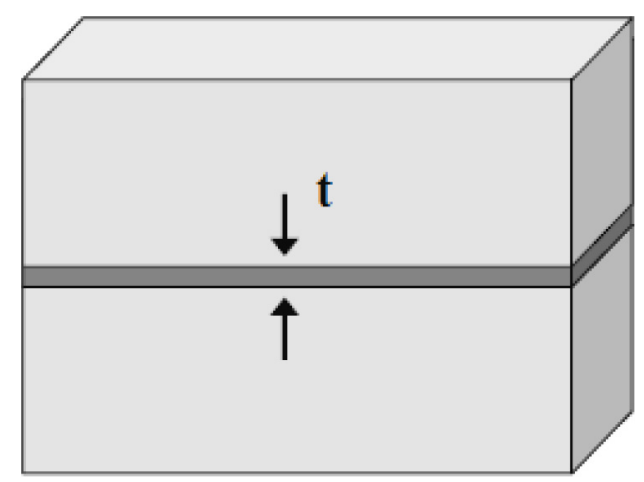

Fig. 1. Schematic of a quantum well of thickness $t$ dividing a bulk semiconductor of larger bandgap.
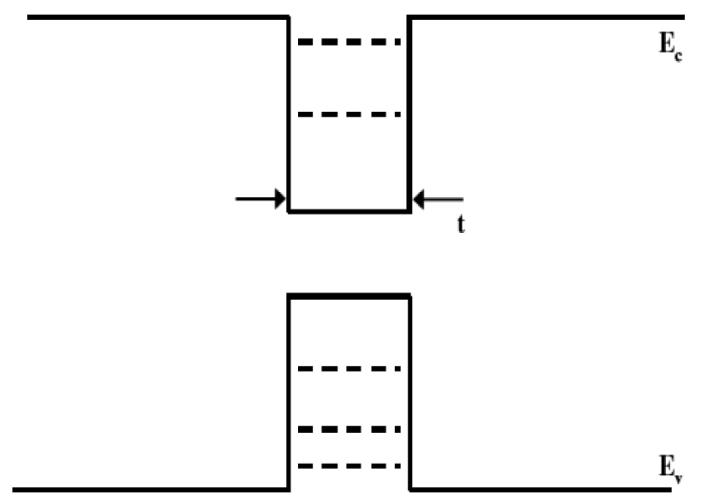

Fig. 2. The corresponding energy band diagram for the schematic shown in Figure 1.

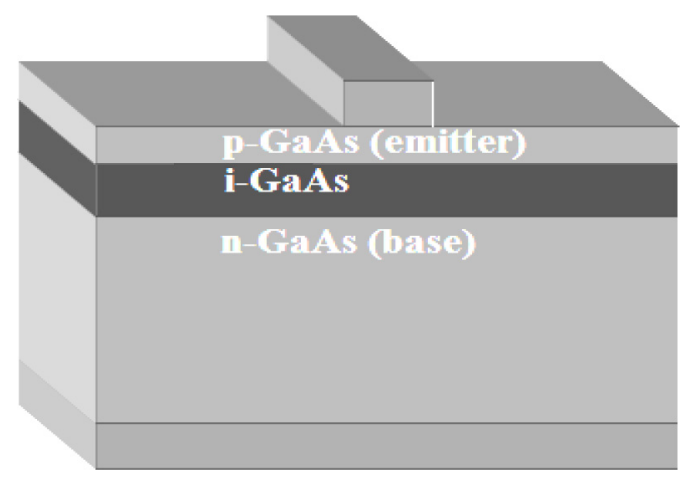

Fig. 3. Simulated GaAs p-i-n solar cell.

\subsection{Method}

The proposed method to increase the efficiency of the device is the incorporation of a nanostructured array in the i-layer. These nanostructures may be quantum wells or quantum dots. A nanostructured array of a material with a smaller bandgap than the host material (that would be GaAs in our case) would allow for additional absorption and photogeneration by light of sub-host-bandgap energy.

Silvaco ATLAS is a $2 \mathrm{D}$ and $3 \mathrm{D}$ device simulator that performs DC, AC, and transient analysis for silicon, binary, ternary, and quaternary material-based devices. ATLAS enables the characterization and optimization of semiconductor devices for a wide range of technologies. Device simulation helps the user to understand and depict the physical processes in a device and also to make reliable predictions of the behavior of the next device generation [9].

Our case study assumes the simulation of an InAs quantum dot GaAs solar cell [3,10]. In this device, an InAs quantum dot array is placed in the i-layer of a GaAs p-i-n cell. The material parameters are presented in Table 1. Unfortunately, ATLAS cannot be used to simulate quantum dots specifically; however, it can be used to simulate quantum wells. Therefore, as an approximation for quantum dots, InAs quantum wells were placed in the ilayer of the experimental device.

\section{Results and discussions}

The code we used for our simulation consists of two ATLAS decks. These decks individually simulate the quantum mechanical effects that are produced due to the inclusion of a nanostructure in a single-junction solar cell:

- first deck is used for analysis of the quantum confined regions and also to determine the properties of the eigenstates located there;

- second deck simulates the electroluminescence occurring due to the nanostructured regions.

\subsection{Property analysis of the eigenstates}

In our case study, an array of $6 \mathrm{~nm}$ InAs quantum wells with 7-nm barrier spacing was placed symmetrically in the ilayer of the p-i-n device. Along with the band diagram of this region, in Figure 4 are displayed the energy eigenvalues arising due to quantization in the wells; accordingly, one electron eigenstate is realized in the conduction band while in the valence band occur five heavy hole eigenstates and one light hole eigenstate. The corresponding eigenfunctions

Table 1. Material parameters for semiconductors used in this work.

\begin{tabular}{lllllllllll}
\hline & $\varepsilon_{\mathrm{r}}$ & $E_{\mathrm{g}}(\mathrm{eV})$ & $\chi(\mathrm{eV})$ & $N_{\mathrm{c}}\left(\mathrm{cm}^{-3}\right)$ & $N_{\mathrm{v}}\left(\mathrm{cm}^{-3}\right)$ & $n_{\mathrm{i}}\left(\mathrm{cm}^{-3}\right)$ & $m_{\mathrm{n}}{ }^{*} / m_{0}$ & $m_{\mathrm{p}}{ }^{*} / m_{0}$ & $\begin{array}{l}A_{\mathrm{n}}{ }^{*} \\
\left(\mathrm{~A} / \mathrm{cm}^{2}-\mathrm{K}^{2}\right)\end{array}$ & $\begin{array}{l}A_{\mathrm{p}}{ }^{*} \\
\left(\mathrm{~A} / \mathrm{cm}^{2}-\mathrm{K}^{2}\right)\end{array}$ \\
\hline GaAs & 13.2 & 1.42 & 4.07 & $4.35 \times 10^{17}$ & $1.29 \times 10^{19}$ & $2.67 \times 10^{6}$ & 0.067 & 0.642 & 6.29 & 105 \\
InAs & 14.6 & 0.35 & 4.67 & $9.33 \times 10^{16}$ & $8.12 \times 10^{18}$ & $1.00 \times 10^{15}$ & 0.024 & 0.471 & 2.88 & 56.6 \\
\hline
\end{tabular}




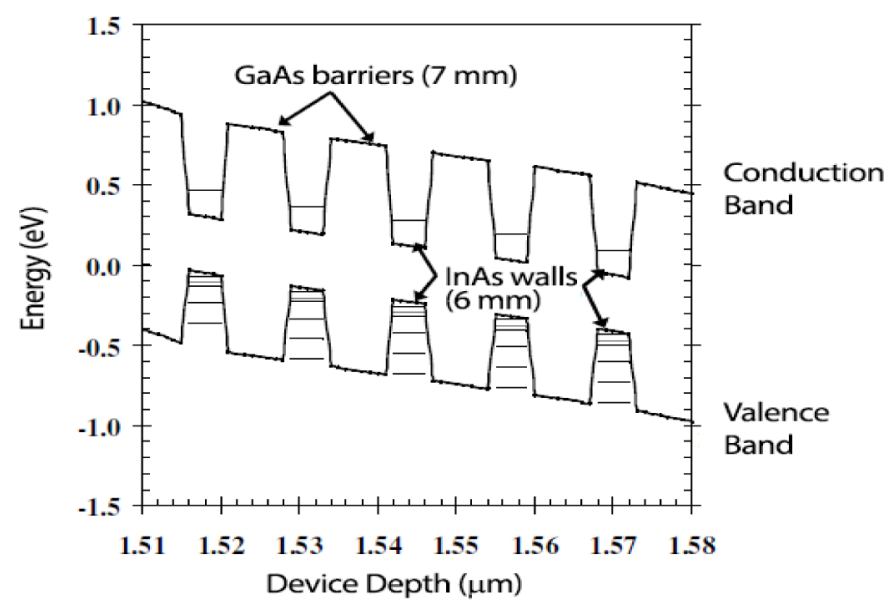

Fig. 4. Band diagrams of InAs quantum wells in the i-layer of the GaAs p-i-n solar cell (energy axis is referenced to the Fermi level).

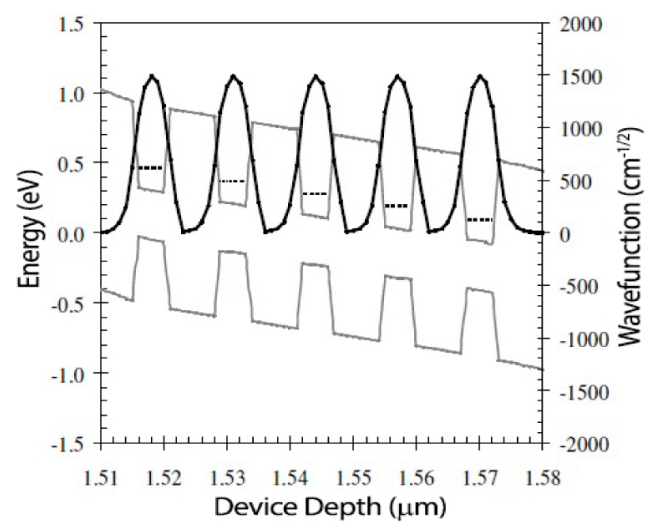

Fig. 5. Eigenfunction overlying the band diagram corresponding to the single conduction electron state.

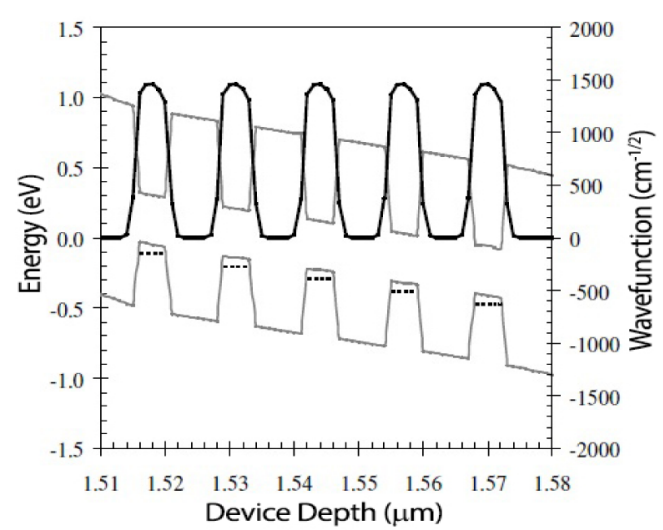

Fig. 6. Eigenfunction overlying the band diagram corresponding the single light hole state.

are plotted in Figure 5 (single conduction electron state), Figure 6 (single light hole state) and Figure 7 (each of the five heavy hole states).

\subsection{Simulation of the electroluminescence}

A standard method for characterizing the quantum dots is to analyze their luminescent emissions. Based on the spontaneous emission model, Figures 8 and 9 display the electroluminescence of the p-i-n device due to varying thicknesses of the InAs wells. The literature indicates an electroluminescence of $\sim 1050 \mathrm{~nm}$ for $6 \mathrm{~nm}$ quantum dots [11].
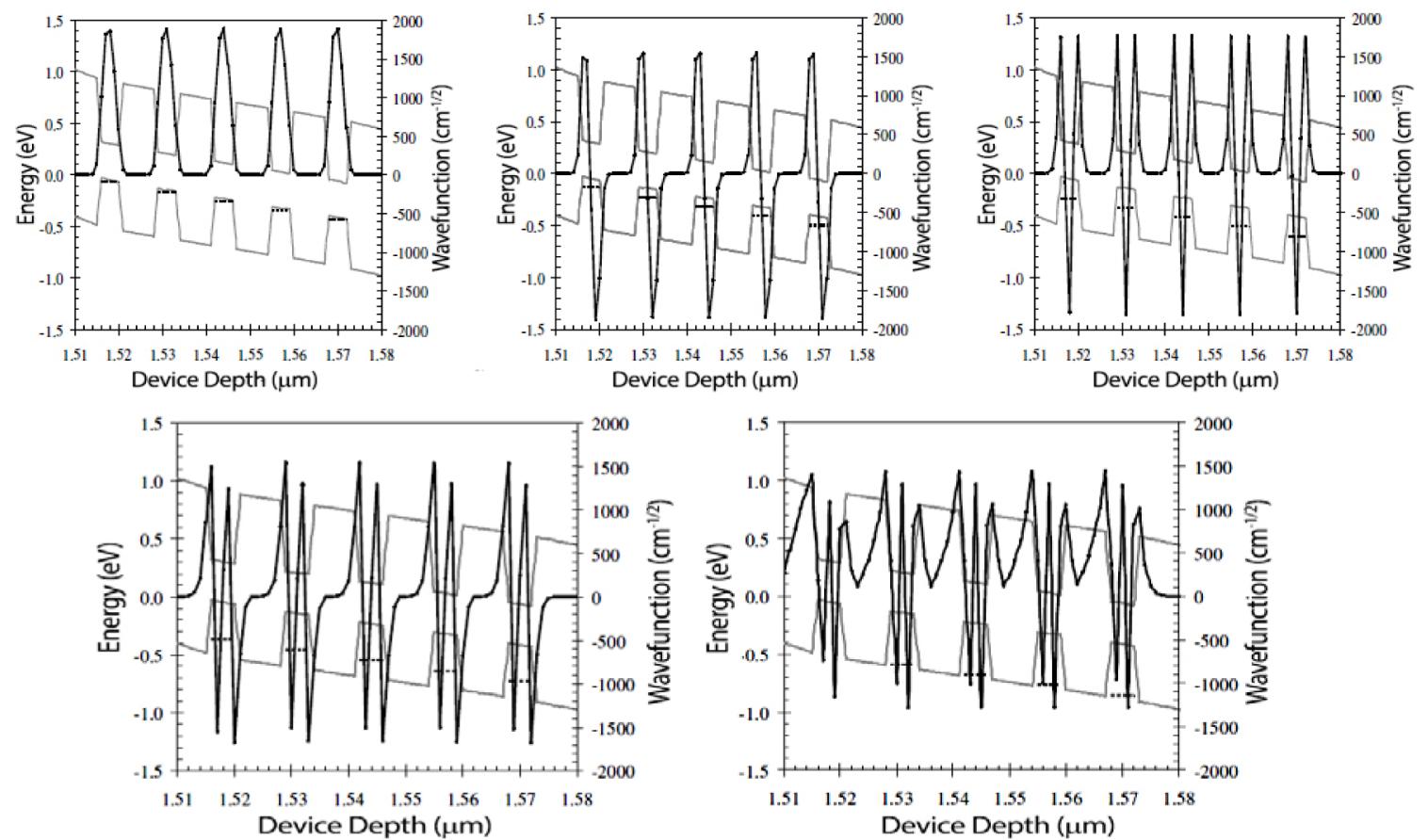

Fig. 7. Eigenfunctions overlying the band diagram corresponding to each of the five heavy hole states. 


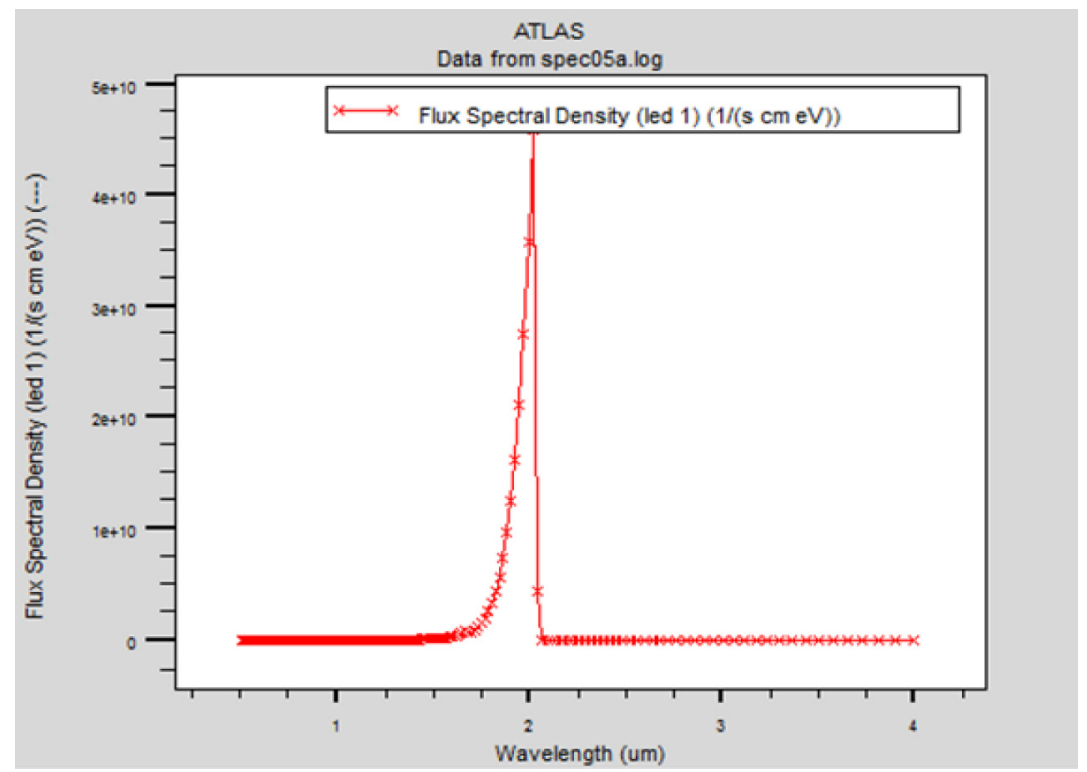

Fig. 8. Simulated electroluminescent spectrum for an InAs well thickness of $6 \mathrm{~nm}$, as displayed in ATLAS.

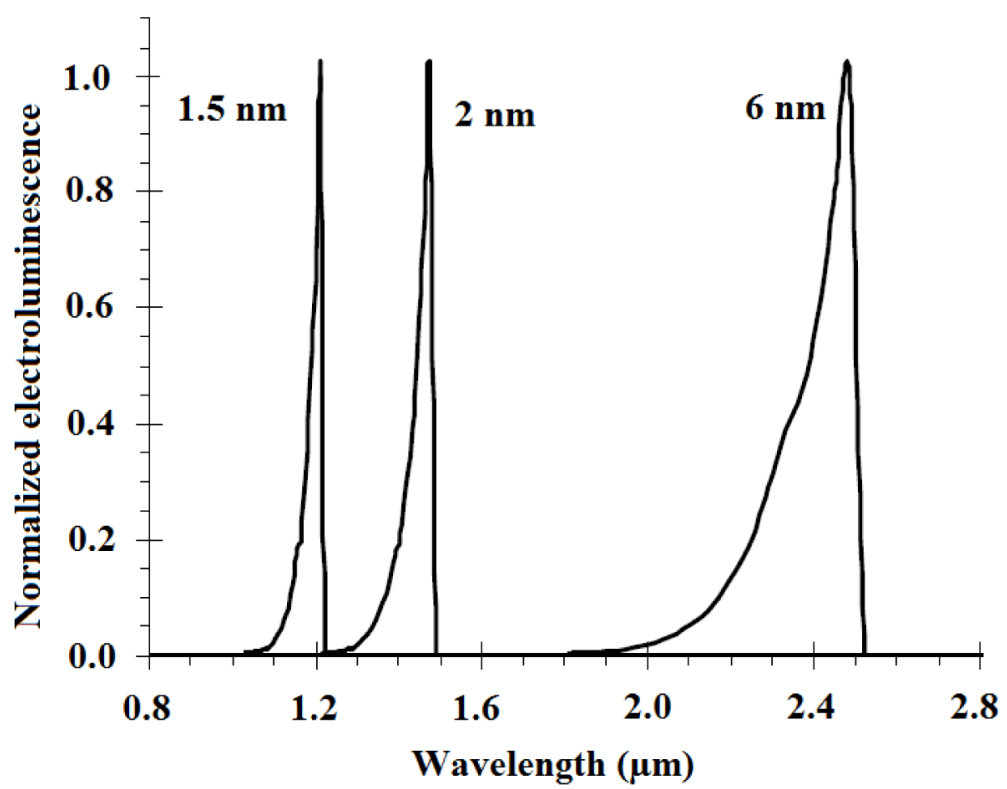

Fig. 9. A comparison of simulated electroluminescent spectra for several different InAs well thicknesses.

\section{Conclusions}

In this paper, we have presented a few modeling solutions necessary for the analysis of the next generation of $\mathrm{PV}$ devices. We have shown that Silvaco ATLAS device simulator is able to simulate basic solar cell performance. A nanostructured solar cell was simulated relying upon the single junction model. A quantum well model (specifically InAs quantum wells being placed in the space charge region of the GaAs device model) was invoked as an approximation to the InAs quantum dot devices currently being investigated. This was a justified approach, as useful results were obtained and presented. Specifically, the properties of the eigenstates that arise due to quantum confinement were obtained giving rise to recommendations on the design of the nanostructured array. Unfortunately, a quantum mechanical model of photoabsorption does not exist in the software package; however, a model of spontaneous emission is present. This made possible the utilization of electroluminescence arising from the quantum confined layers as another possible tool for characterizing the quantum dots. 
We hope this work could serve as a guide to the next series of modeling and simulation efforts that will help in the development of novel photovoltaic devices.

\section{References}

1. S.M. Hubbard, C. Cress, C. Bailey, R. Raffaelle, S. Bailey, D. Wilt, Effect of Strain Compensation on Quantum Dot Enhanced GaAs, Solar Cells Appl. Phys. Lett. 92, 123512 (2008)

2. S.M. Sze, K. Ng, Physics of semiconductor devices, 3rd ed. (John Wiley \& Sons, Hoboken, 2007) p. 58

3. R. Aguinaldo, Modeling solutions and simulations for advanced III-V photovoltaics based on nanostructures (Rochester Institute of Technology, Rochester, 2008) p. 10

4. S. Fara, P. Sterian, L. Fara, M. Iancu, Influence of the optical parameters of the quantum well solar cells upon conversion efficiency, J. Optoelectron. Adv. M. 12, 129 (2010)
5. L. Fara, M. Yamaguchi, Advanced solar cell materials, technology, modeling, and simulation (IGI Global, Hershey, 2013) p. 48

6. E. Yablonovitch, O.D. Miller, S.R. Kurtz, in 2012 38th IEEE Photovoltaic Specialists Conference, 001556 (2012)

7. X. Wang, M.R. Khan, J. Gray, M.A. Alam, M. Lundstrom, Design of GaAs Solar Cells Operating Close to the Shockley Queisser Limit, IEEE J. Photovolt. 3, 737 (2013)

8. S. Fara, P. Sterian, L. Fara, M. Iancu, A. Sterian, New results in optical modelling of quantum well solar cells, Int. J. Photoenergy 2012, (2012)

9. http://www.silvaco.com/products/tcad/device_simulation/ atlas/atlas.html

10. R. Raffaelle, S. Sinharoy, J. Andersen, D. Wilt, S. Bailey, Multi-Junction Solar Cell Spectra Tuning with Quantum Dots Conf. Rec., in 2006 IEEE 4th World Conf. Photovoltaic Energy Conv. (2006), pp. 162-166

11. S.M. Hubbard, R.P. Raffaelle, R. Robinson, C. Bailey, D. Wilt, D. Wolford, W. Maurer, S. Bailey, Growth and characterization of InAs Quantum Dot Enhanced Photovoltaic Devices, Mater. Res. Soc. Symp. Proc. 1017E, DD13 (2007)

Cite this article as: A. Badea, F. Dragan, L. Fara, P. Sterian, Quantum mechanical effects analysis of nanostructured solar cell models, Renew. Energy Environ. Sustain. 1, 3 (2016) 